\section{Malthus with a computer}

Malthus with a computer is the provocative title of a critical series of essays on the computer models described in the much-publicized book The Limits to Growth ${ }^{1}$ that Europhysics News reported upon in July $1972^{2}$. First published ${ }^{3}$ as the February 1973 issue of Futures, Malthus with a computer is the response of the professional Science Policy Research Unit (SPRU) at the University of Sussex, UK, to what they consider to be over-exaggerated claims and a less than rigorous approach to forecasting. The series is introduced by C. Freeman, Director of SPRU, who, from the outset, makes it clear that he is in complete agreement with the urgency of many of the social problems studied by Meadows et al at MIT and formulated by the sponsors, the Club of Rome, as the World Problematique.

However, the SPRU team believe that computer model-building techniques and systems dynamics have serious limitations and are in danger of misuse. Each of the sub-systems of the MIT model (resources, population, agriculture, capital and pollution) are critically analyzed and, apart from the pollution sub-system, are unsatisfactory. Also, the model as a whole has sensitivity to a few key assumptions that is difficult to justify. Apparently, the MIT models do not even match real world data for 1900-1970, the theoretical structure is over-elaborate, and the assumptions do not pay sufficient attention to economics and sociology. A major criticism is that computer modelling gives the impression of being neutral, whereas it has a distinct political bias and has cer-

\section{Controlled Fusion and \\ Plasma Physics Proceedings}

The Sixth European Conference on Controlled Fusion and Plasma Physics will be held in Moscow from 30 July 3 August 1973. The Conference Proceedings will contain 150 original papers and will be published in English in July 1973. The volume of 18 review lectures will be in English and issued at the beginning of 1974 .

If orders are placed for the Proceedings before 1 July 1973, they will be guaranteed; if placed afterwards, they will be fulfilled if possible, since only a small number of extra copies, above the participants' requirements, will be printed. Orders may be placed with M. Rabinovich, P.N. Lebedev Institute, Leninsky Prospekt 53, Moscow B-312. tain implicit values. The SPRU team maintain that the MIT assumptions are heavily influenced by contemporary social theories and values to which the computer modellers have been exposed.

The Sussex University physicist, R. Golub, reflects the disquiet that many other physicists have expressed concerning the self-delusions arising from the MIT computer model-building :

(a) spurious appearance of precise knowledge of quantities and relationships which are unknown and in many cases unknowable ;

(b) neglect of factors which are difficult to quantify such as policy changes or value changes ;

(c) gross over-simplification because of the problem of aggregation and comparative simplicity of computers and mathematical techniques ;

(d) tendency to treat some features of the model as rigid and immutable ;

(e) difficulty for non-numerate or those lacking computer access to rebut essentially tendentious and rather naive political assumptions.

In a more positive vein, the SPRU team acknowledge the importance of the Meadows' book as a stimulus to future work. Also, as Freeman writes the MIT work has done a great deal in compelling social scientists to reexamine some of their assumptions and in exposing the limitations both of data and of satisfactory explanatory theories for some of the most important social mechanisms'. Freeman also states that he personally believes that the open public debate surrounding the MIT work is their most important achievement, and he agrees with their important emphasis on delays in governmental responses to the urgent problems of deployment of scientific resources today.

Despite the technical criticisms and the exposed political bias of The Limits to Growth, it remains a turningpoint in the field of socio-economic theory which has now captured public attention.

\section{References}

'Meadows, D., et al, Technical Report and The Limits to Growth (Universe Books, New York, and Earth Island, London, 1972) ${ }^{2}$ World Problematique, Europhysics News, 36 (July 1972) 4,5

${ }^{3}$ Malthus with a computer (Series of critical essays in Futures (February 1973) (IPC Science and Technology Press, Guildford, UK, 1973)
New Books from North-Holland

\section{Fast Ion Transport in Solids, Solid State Batteries and Devices}

Proceedings of the NATO Sponsored Advanced Study Institute, Belgirate, Italy, 5-15 September 1972

Edited by W. VAN GOOL, University of Utrecht, The Netherlands

1973. approx. 700 pages

Dfl. 130.00 (about $\$ 45.60$ )

The Proceedings of the 10-day Summerschool on Fast ion transport in solids solid state batteries and devices contain a thorough discussion of the diffusion phenomena in solids. The present state of the theory of anomalously fast diffusion (super ionic conductors) is discussed. Both research and teaching contributions deal with known and new solid electrolyte materials and battery systems.

Teaching contributions by: A. Ascoli, J.W. Geus, W. van Gool, Y. Haven, L. Heyne, J.M. Honig, R.A. Huggins, M. Jozefowicz, R. Kikuchi, A.D. Le Claire, C.C. Liang, A. Lundén, M.E. Milberg, S.P. Mitoff, M. O'Keeffe, B.B. Owens, S. Pizzini, D.O. Raleigh, H. Rickert, R.S. Roth, B.C.H. Steele, J.B. Wagner, Jr., I. Wynn Jones

\section{Few Particle Problems in Nuclear Interaction}

Proceedings of the International Conference on Few Particle Problems in Nuclear Interaction, Los Angeles, August, 1972.

Edited by I. SLAUS, S.A. MOSZKOWSKI R.P. HADDOCK and W.T.H. VAN OERS

1973. approx. 1100 pages Dfl. 160.00 (about $\$ 56.10$ )

Presents and authoritative and up-to-date review of recent developments in low and medium energy few particle problems. Special emphasis is given to the nuclear three body problem, nuclear forces (in particular the three body force), symmetries, and the interaction of mesons. leptons and photons with few nucleon systems. Applications of these results to astrophysics and nuclear structure studies are summarized.

\section{NORTH}

P.O. Box 211 - Amsterdam - The Netherlands Distributed in the United States and Canada by American Elsevier Publishing Company, Inc., 52 Vanderbilt Avenue, New York, N.Y. 10017 